\title{
HYSTERETIC BEHAVIOR OF SHEAR PANEL DAMPERS UNDER HIGH AXIAL COMPRESSION LOADING
}

\author{
Zhiyi Chen ${ }^{1, * 2,3}$, Guoqiang Bian $^{3}$ and Yu Huang ${ }^{3,4}$ \\ ${ }^{I}$ Associate Professor, Key Laboratory of Geotechnical and Underground Engineering of \\ the Ministry of Education, Tongji University, Shanghai 200092, China \\ ${ }^{2}$ State Key Laboratory of Geo-Hazard Prevention and Geo-Environment Protection, \\ Chengdu University of Technology, Chengdu 610059, China \\ ${ }^{3}$ Department of Geotechnical Engineering, Tongji University, Shanghai 200092, China \\ ${ }^{4}$ Professor, Key Laboratory of Geotechnical and Underground Engineering of the Ministry of Education, \\ Tongji University, Shanghai 200092, China \\ *(Corresponding author: E-mail: zhiyichen@tongji.edu.cn)
}

Received: 30 October 2011; Revised: 31 March 2012; Accepted: 12 April 2012

\begin{abstract}
This paper aims to investigate the hysteretic behavior of shear panel dampers (SPDs) made of mild steel Q235B incorporated in underground structures against earthquakes. Five SPDs were given cyclic shear loading tests, with the axial compression ratio and the web slenderness as test variables. The shear bearing capacity and the ductility decreased continually with increasing axial compression ratio. When the axial compression ratio exceeded 0.6 , the hysteretic behavior was seriously affected. Cumulative energy dissipated by the test specimen with an axial compression ratio of 0.7 was only half that of the one with a ratio of 0.3 . The control group (the SPD without the web) was set to estimate the horizontal resistance of upper and lower flanges that were mostly induced by flexure. The contribution of two flanges in resisting the shear deformation was about $15 \%$.
\end{abstract}

Keywords: Shear panel dampers, Axial compression ratio, Hysteretic behavior, Energy dissipation, Underground structures

\section{INTRODUCTION}

The seismic response and disaster mechanism of underground structures have received increasing attention in theory, numerical simulation and model testing during strong earthquakes. However, studies have failed to involve direct measures or the principle method of seismic damage mitigation for underground structures. For underground structures with large span or complex style, dynamic effects emerge inspired by seismic environment due to its larger scale and fewer soil constraints. Traditional static simplified methods cannot meet requirements for the analysis and design of large span underground structures because dynamic characteristics caused by ground deformation must be considered during an earthquake. Traditional seismic methods, such as increasing section area and reinforcement ratio, will increase the rigidity of underground structures and enhance their internal forces. Meanwhile, such a partial reinforcement approach cannot make a good match to the stiffness and ductility, and will easily lead to stiffness mutation, local stress concentration and weak regional metastasis, which cannot improve the seismic performance of the whole structure.

As a passive control method of structural seismic control, structural seismic energy dissipation technology has had great development and wide application. This technology sets up energy dissipation devices in parts of a structure. The friction and deformation of the device's material reduces the seismic response of the main structure and avoids its damage or collapse. A shear panel damper (SPD) is a passive control device that uses the shear hysteresis of the metal plate as the source of energy dissipation. Such dampers are known to enter the plastic state before the main structure and possess energy dissipation capacity, which makes them cost-effective in ground buildings and bridges. When Nakashima used low-yield steel as a damper material, a test indicated that the SPD had excellent hysteretic behavior and strain-hardening was very conspicuous under cycles with increasing deformation [1,2]. Nakashima also proposed simple models that could 
simulate the hysteretic behavior of shear panels [3]. The results of an SPD frame-structure test done by Chen and Kuo [4] showed that SPDs could effectively dissipate seismic energy during an earthquake and control the main frame structure in the elastic range. Chen et al. [5] studied seismic energy absorption effects of SPDs in a steel bridge structure using numerical simulation. The results showed that the top displacement of bridge piers was reduced to $1 / 2 \sim 1 / 4$, and the effective strain ratio of the bottom of the pier dropped to 0.9 to 3.3 from 23 , which was compared with the structure without dampers.

For the ground structure, shear panel dampers mainly resist lateral earthquake loading, which passes only a little vertical force from the beam to the damper. In designing hysteretic tests of shear panel dampers, vertical axial force is often ignored, and only horizontal shear cyclic loading is applied. However for shear panel dampers to be used in underground structures, it is different. Figure 1(a) illustrates an SPD being replaced in the central column of an underground subway station. As shown in Figure 1(b), the underground structure is racking under horizontal earthquake loading. The SPD deforms in shear and dissipates seismic energy through metal plasticity, being similar to be in the ground structure. However, the failure modes of the central columns in the Daikai subway station, which was a famous and well-documented case for underground structures damaged and collapsed during the 1995 Kobe earthquake, suggested that their collapse was mainly due to overburdened soil and earthquake-induced acceleration. Existing experimental research indicates that a high axial compression ratio decreased the drift capacity of the RC column [6,7]. The soil-structure FEM analysis [7] showed a variation between approximately 1800 and $4800 \mathrm{kN}$ axial load induced in the central column, which were about as high as $30 \%$ and $69 \%$ of the designed axial strength capacity of the central column $(7000 \mathrm{kN})$. Therefore even if post-installation method is used to minimize potential axial compression in SPD, the large axial forces cannot be avoided during application. Recent research [8] on shear beam links (its configuration is somewhat like two or three side-by-side SPDs) in eccentrically braced frames revealed that axial forces would affect the overstrength, result in early flange yielding accompanied with web yielding. Consequently the effect of a high axial compression ratio should be fully accounted for.

$$
\text { Ground surface }
$$

(a)
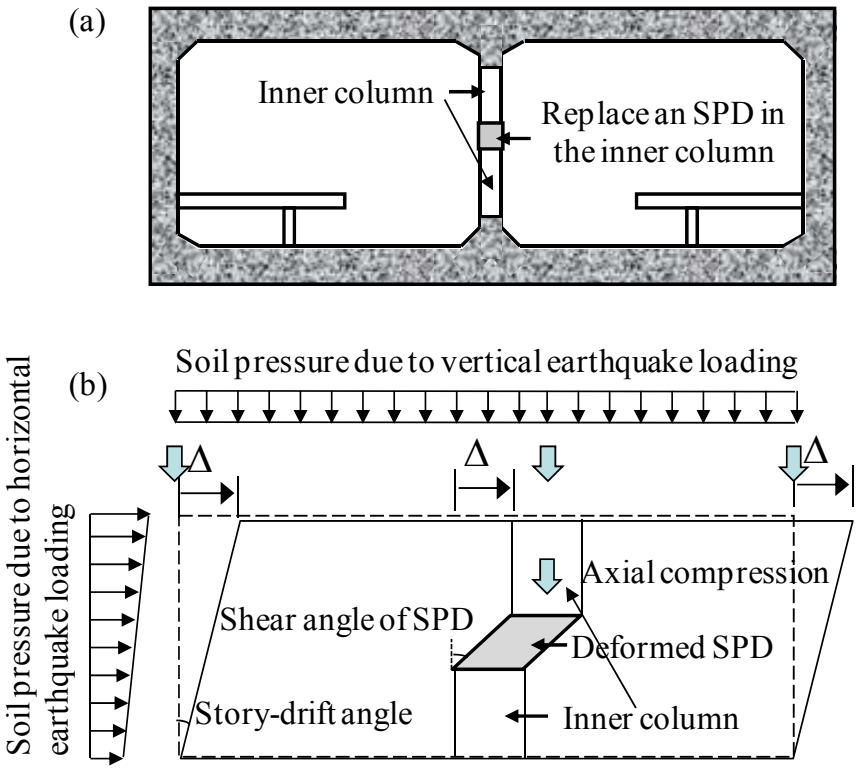

Figure 1. Schematic Design of a Shear Panel Damper in Underground Structures 
In view of this, a series of cyclic shear tests was conducted on SPDs with different axial compression ratios. The main purpose was to quantify their significant hysteretic behavior and energy dissipation capacity, because such quantification is of great importance in estimating seismic damage mitigation by hysteretic dampers.

\section{TEST PROGRAM}

\section{$2.1 \quad$ Test Specimens}

The SPD specimens are shown in Figure 2. Each specimen consists of a web (shear panel), two flanges, stiffeners and two loading plates, which are made of mild steel Q235B. The clear dimension of each panel is $400 \mathrm{~mm}(\mathrm{~W}) \times 400 \mathrm{~mm}(\mathrm{H})$, and the thicknesses are $5 \mathrm{~mm}$ and $6 \mathrm{~mm}$. Vertical flange thicknesses were $18 \mathrm{~mm}$ and $24 \mathrm{~mm}$, respectively, and the width was $120 \mathrm{~mm}$. The loading plates were $450 \mathrm{~mm} \times 400 \mathrm{~mm}$ (upper) and $880 \mathrm{~mm} \times 400 \mathrm{~mm}$ (lower) respectively, and their thickness was $20 \mathrm{~mm}$. Stiffeners were $40 \mathrm{~mm}$ in width and $6 \mathrm{~mm}$ in thickness. The panel was fillet-welded all around to the flanges and loading plates.

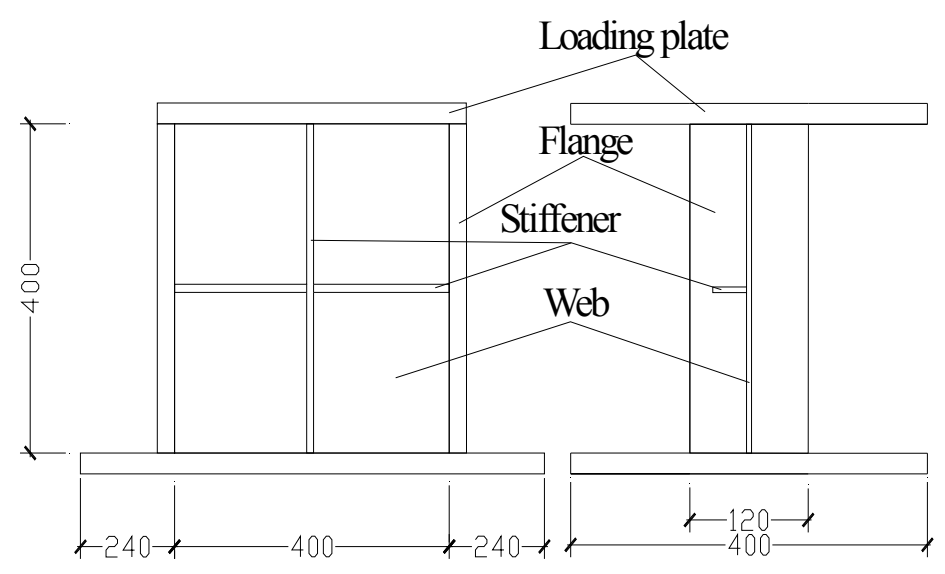

Figure 2. Dimensions of Test Specimen (Unit: mm)

Mild steel Q235B is used as the material of a stiffened SPD. An SPD is characterized by the web slenderness parameter, $R_{w}$, defined as [9]

$R_{w}=\frac{B_{w}}{T_{w}} \sqrt{\frac{12\left(1-v^{2}\right) \tau_{y}}{k_{s} \pi^{2} E}}$

where $B_{w}$ and $T_{w}=$ width and thickness of web respectively, $v=$ Poisson's ratio, $\tau_{y}=$ shear yield of web material, $E=$ Young's modulus of elasticity, and $k_{s}=$ elastic buckling coefficient of a simply-supported plate under shear.

A total of six specimens were tested. These included SPDs (Rw04-z05, Rw03-z03, Rw03-z05, Rw03-z06, Rw03-z07) and the control specimen (Flange). The specimen parameters are shown in Table 1. The meaning of the specimen name, for instance Rw03-z03, is $R_{w}=0.3$ and axial compression ratio $=0.3$. The control specimen "Flange" had only surrounding members, namely, flanges and loading plates without the web and the stiffeners. 
Table 1. Summary of Test Specimens

\begin{tabular}{|c|c|c|c|c|c|c|c|c|c|c|}
\hline$z$ & Specimen & $R_{w}$ & $B_{w}(\mathrm{~mm})$ & $T_{w}(\mathrm{~mm})$ & $T_{f}(\mathrm{~mm})$ & $B_{f}(\mathrm{~mm})$ & $T_{s}(\mathrm{~mm})$ & $B_{s}(\mathrm{~mm})$ & $A\left(\mathrm{~mm}^{2}\right)$ & $P(\mathrm{kN})$ \\
\hline 0.7 & Rw03-z07 & 0.3 & 400 & 6 & 24 & 120 & 6 & 40 & 8400 & 1381.8 \\
\hline 0.6 & Rw03-z06 & 0.3 & 400 & 6 & 24 & 120 & 6 & 40 & 8400 & 1185 \\
\hline \multirow{3}{*}{0.5} & Rw04-z05 & 0.4 & 400 & 5 & 18 & 120 & 6 & 40 & 6560 & 770.8 \\
\hline & & & & & & & & & & \\
\hline & Rw03-z05 & 0.3 & 400 & 6 & 24 & 120 & 6 & 40 & 8400 & 987 \\
\hline 0.3 & Rw03-z03 & 0.3 & 400 & 6 & 24 & 120 & 6 & 40 & 8400 & 600 \\
\hline 0.0 & Flange & 0.3 & - & - & 24 & 120 & - & - & - & 0 \\
\hline
\end{tabular}

Previous researches $[9,10,11]$ have shown that the suitable range of web slenderness parameter $R_{w}$ is $0.2-0.5$ for shear panel dampers. Of these, the values of 0.3 and 0.4 are more flexible for practical design purposes. Therefore, the web slenderness parameter $R_{w}$ was taken as 0.3 and 0.4 in the present experiment. The axial compression ratio was set as the most important parameter to study the hysteretic behavior of SPDs under high axial compression. To find the critical axial compression ratio, a series of axial compression ratios $(0.3,0.4,0.5,0.6$ and 0.7$)$ was set. The axial compression ratio is defined as the ratio of axial force to yield axial force of the SPD. In addition, a control specimen was set up to understand the effect of flanges of an SPD on the shear bearing capacity and energy dissipation performance.

The photos of typical SPD and "Flange" specimens are shown in Figure 3 and Figure 4, respectively.

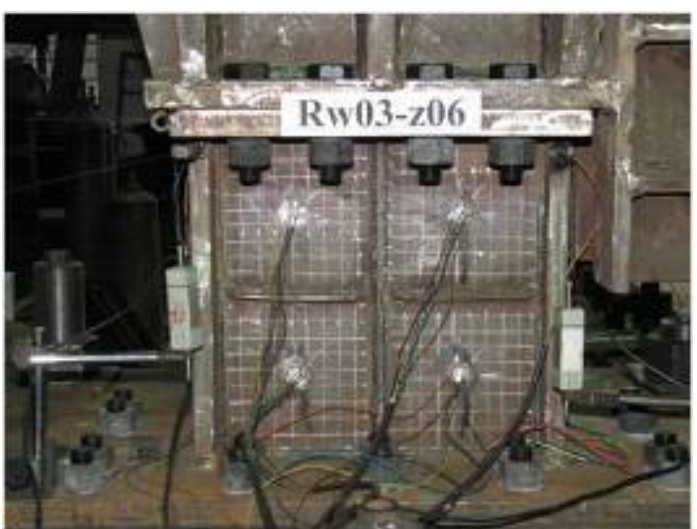

Figure 3. Stiffened Shear Panel Dampers

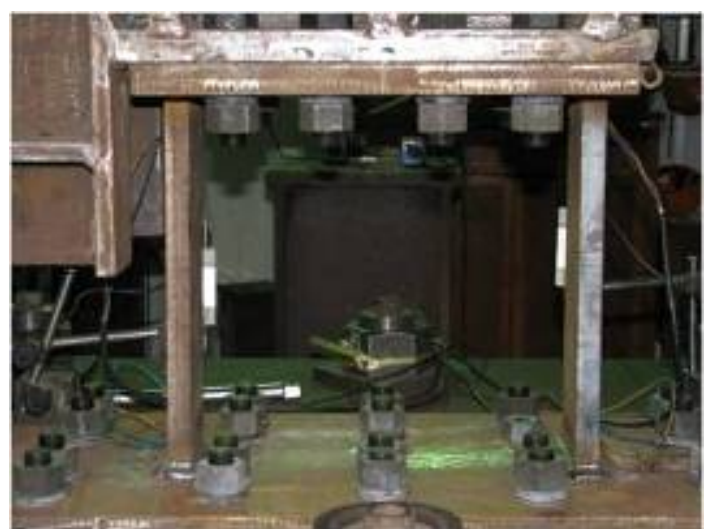

Figure 4. Control Specimen (Flange)

\section{$2.2 \quad$ Test Setup}

Figure 5 shows the loading setup employed in the test. A $10,000 \mathrm{kN}$ multi-functional structure testing machine in Tongji University was used which can apply force and displacement loading by the horizontal and vertical actuators simultaneously. Labels 1, 2, 3 and 4 are loading head, lateral connection, base, and test specimen, respectively. The loading head transfers the axial loading and 
cyclic shear loading to the test specimen; the lateral connection is used to connect the horizontal actuator and the loading head; the base is used to fix the test specimen with anchor bolts.

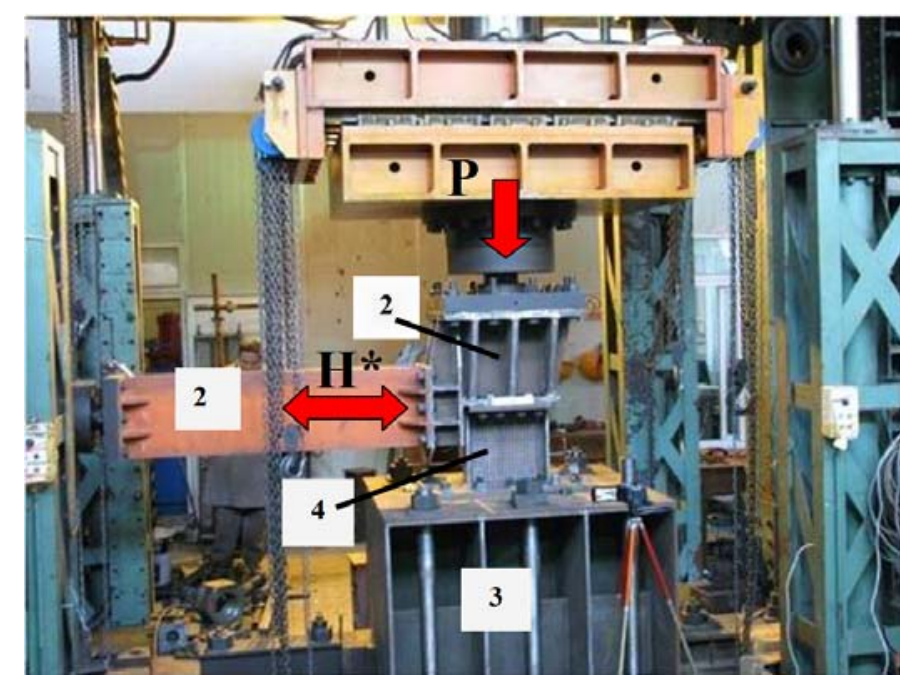

Figure 5. Loading Setup in Test

\subsection{Loading Program and Measurement}

The test machine had four actuators: the vertical main actuator, the first-level actuator, the second-level actuator, and the supporting vertical actuator. The displacement and force of the four actuators was monitored by digital displacement and force transducers. The axial loading was applied by the vertical main actuator, and the horizontal loading was applied by the second-level actuator. The maximum horizontal thrust was 150 tons, the maximum horizontal pull was 100 tons, and the maximum stroke of the actuator was $\pm 400 \mathrm{~mm}$.

The axial force was loaded onto each specimen listed in Table 1, and then the horizontal cyclic shear loading was applied to the specimen by controlling the displacement at the top relative to its bottom. The value of the horizontal cyclic shear loading, as denoted by $H^{*}$ in Figure 5, was monitored by the load cell attached to the second-level actuator of the test machine.

Displacement transducers and strain gauges were arranged as shown in Figures 6 and 7. The horizontal displacement at the top of the specimen relative to its bottom was measured by a digital displacement transducer (1). A pair of displacement transducers (2 and 3) was used to measure the vertical displacement at the top of the specimen relative to its bottom. Four triaxial strain gauges were glued at the center of each subpanel which was divided by transverse and longitudinal stiffeners, and eight uniaxial gauges were pasted on the surfaces of the flanges, with four gauges on the exterior surface of each flange.

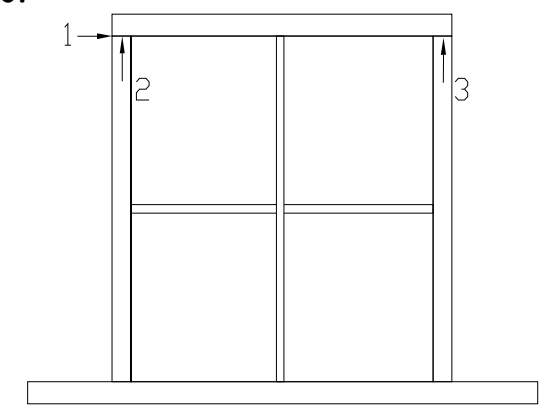

Figure 6. Arrangement of Displacement Transducers 

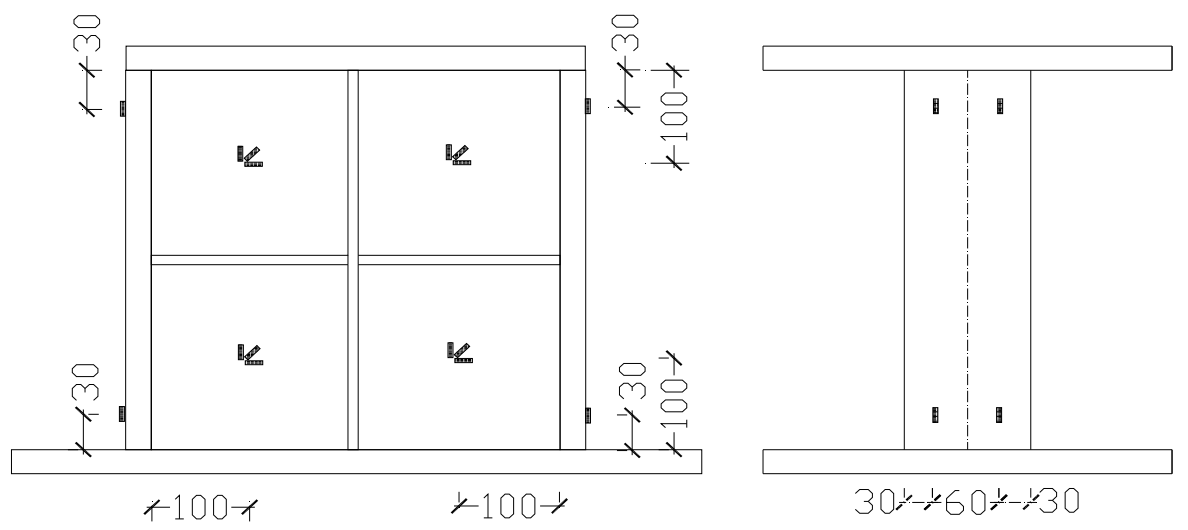

(Unitし mm)

Figure 7. Arrangement of Strain Gauges

\section{TEST PROCESS AND RESULTS}

\subsection{Material Properties}

The results of material tension tests on Q235B mild steel (6 $\mathrm{mm}$ and $24 \mathrm{~mm}$ in thickness), which were used for SPDs in the test, are summarized in Table 2. The yield stress and ultimate stress of the $6 \mathrm{~mm}$ thick plate is about $10 \%$ larger than those of the $24 \mathrm{~mm}$ thick plate.

Table 2. Material Properties

\begin{tabular}{cccccc}
\hline Material & Thickness & $\begin{array}{c}\text { Yield } \\
\text { stress } \\
(\mathrm{MPa})\end{array}$ & $\begin{array}{c}\text { Ultimate } \\
\text { stress } \\
(\mathrm{MPa})\end{array}$ & $\begin{array}{c}\text { Rupture } \\
\text { strain } \\
(\%)\end{array}$ & $\begin{array}{c}\text { Young's } \\
\text { modulus } \\
(\mathrm{GPa})\end{array}$ \\
\hline Q235B & 6.0 & 265.1 & 400.8 & 44.0 & 195.6 \\
Q235B & 24.0 & 239.3 & 360.1 & 43.1 & 202.9 \\
\hline
\end{tabular}

\subsection{Test Process and Test Phenomenon}

The typical damage process in cyclic shear loading can be described as follows: the start of loading, initial web buckling, obvious web buckling, tiny cracks, cracks developing and finally the web failure. The typical failure trend is shown in Figure 8.

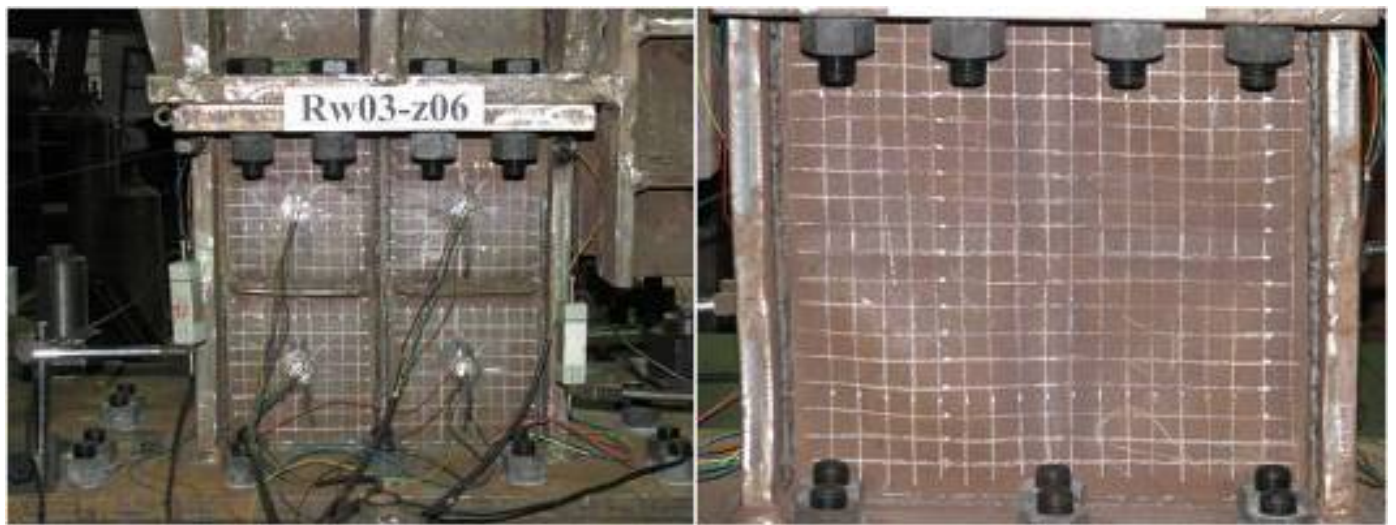

(a) Starting (b) Initial Web Buckling 


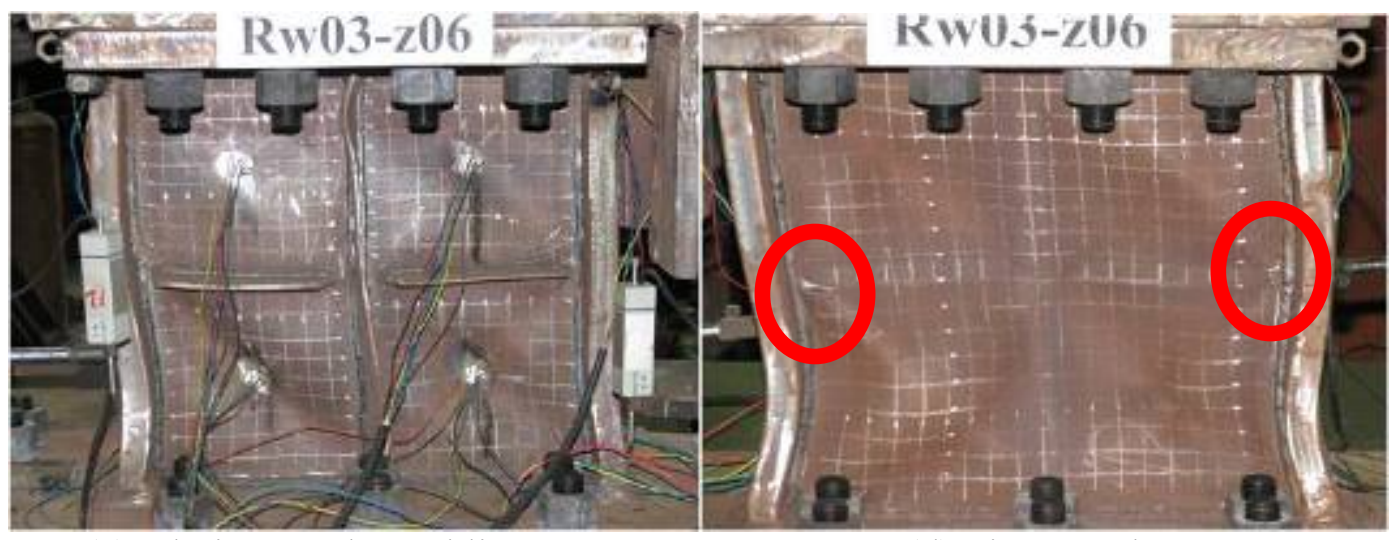

(c) Obvious Web Buckling

(d) Tiny Cracks

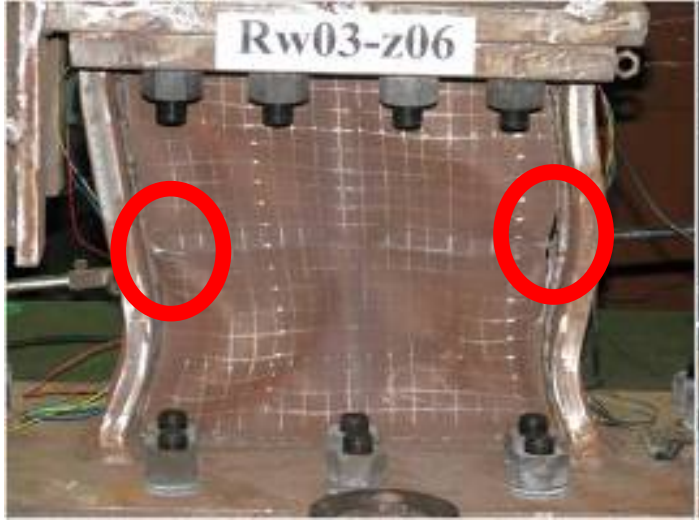

(e) Cracks Developing

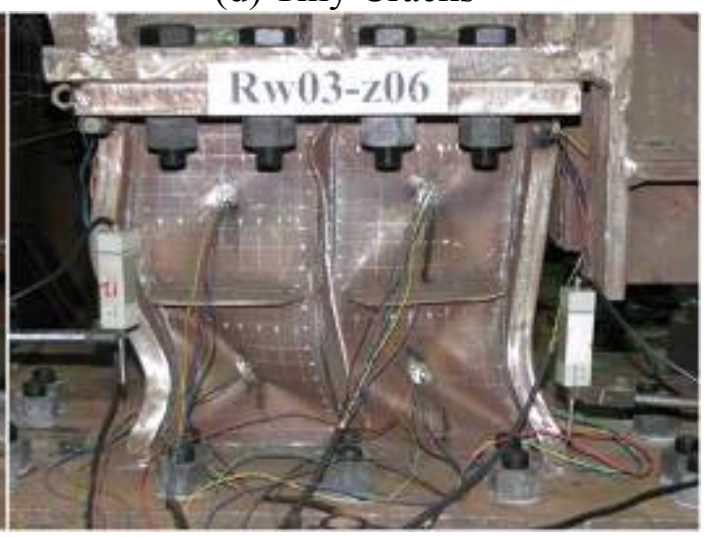

(f) Failure of Test Specimen

Figure 8. Damage Process of SPD under Cyclic Shear Loading

Shear strength increased steadily with increasing shear angle, and reached its peak when obvious web buckling occurred. After keeping smooth for several further loading cycles, shear strength began to decrease as tiny cracks appeared. Soon cracks began to expand and the number of cracks increased, and shear strength quickly degraded. After the web of the SPD was destroyed, flanges could sustain some shear force, but the shear strength quickly degenerated to zero under high axial compression ratio.

The cracks appeared mainly in welded zones between the web, flange and stiffener and local zones with large out-of-plane deformation. The damage details are shown in Figure 9.

\subsection{Horizontal Force versus Shear Angle Relationships}

Hysteretic behavior is of the utmost importance in evaluating the performance of SPDs as energy dissipation devices. The horizontal displacement at the top of the specimen relative to its bottom was measured by digital displacement transducer 1 (see Figure 6). The horizontal force, $H^{*}$, was monitored by the load cell attached to the horizontal actuator (the second-level actuator of the test machine in Figure 5). $H_{y}$ is the yield shear force, = given as the product of the horizontal cross-sectional area of the SPD not including the cross-sectional areas of flanges $\left(B_{w}\right.$ and $T_{w}$ are shown in Table 1$)$, and the yield stress $(265.1 \mathrm{MPa})$ divided by $\sqrt{3}$. The vertical displacement of the two flanges of each specimen, which was measured by transducers 2 and 3 (Figure 6) remained nearly zero before the failure of the test specimen. As a result, the shear angle, $\gamma$, can be calculated by the horizontal displacement $\Delta$ divided by the height of the specimen $(0.4 \mathrm{~m})$. 


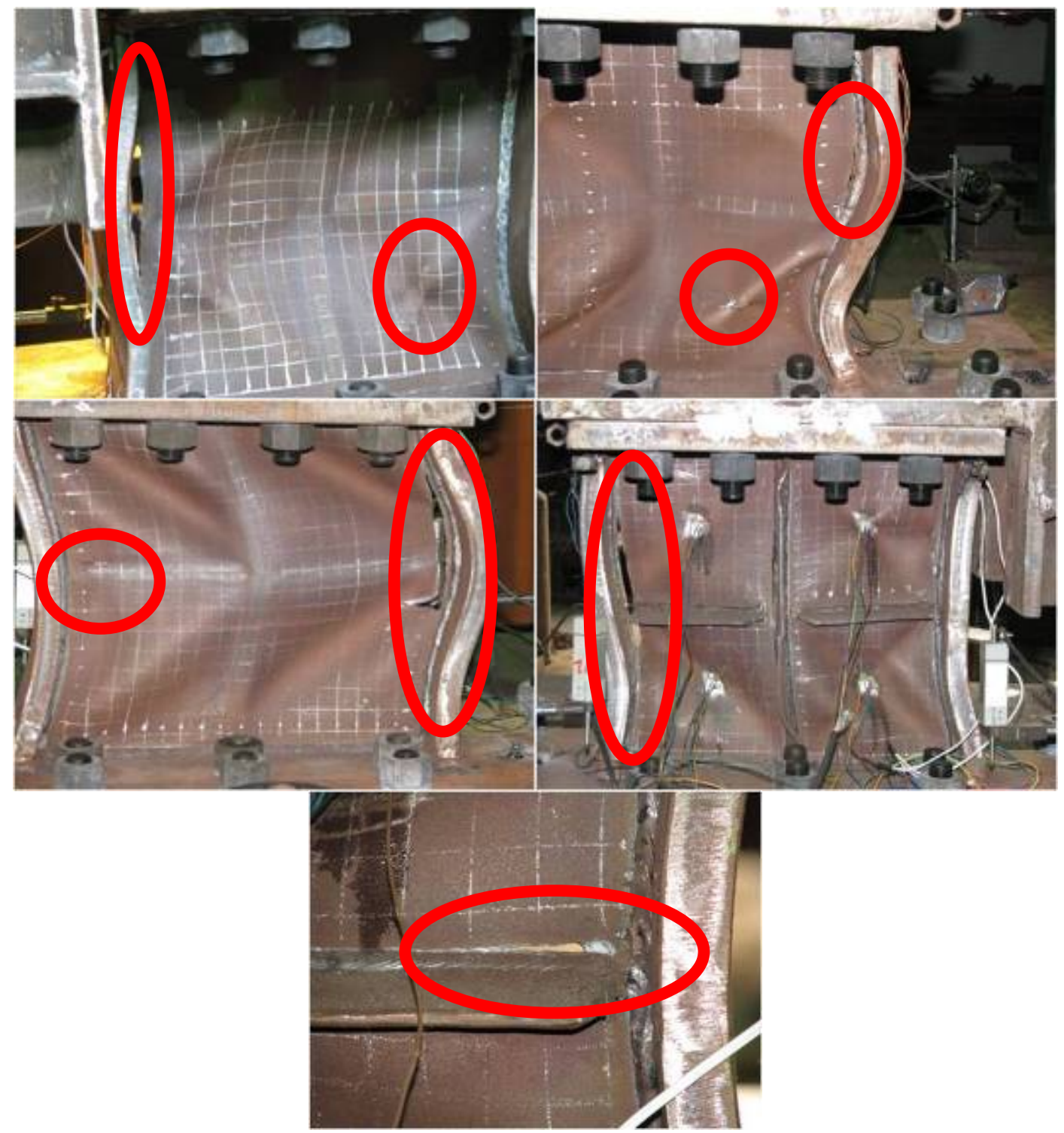

Figure 9. Crack Details

Figure 10 shows the relationship between the normalized horizontal force $\left(H^{*} / H_{y}\right)$ and the shear angle $\gamma$ obtained from the cyclic shear loading test. Yielding can be defined as an apparent departure from the initial linear relationship. It started at a normalized horizontal force of 1.25 (Rw04-z05), 1.21 (Rw03-z03), 1.16 (Rw03-z05), 1.12 (Rw03-z06), 1.01 (Rw03-z07). Comparing the test specimens Rw03-z03, Rw03-z05, Rw03-z06 and Rw03-z07 shows that the normalized horizontal force continually decreased with the increasing axial compression ratio, especially for the high axial compression ratio of 0.7 . The horizontal force increased steadily after yielding and reached 1.83 (Rw04-z05), 1.68 (Rw03-z03), 1.70 (Rw03-z05), 1.74 (Rw03-z06), and 1.66 (Rw03-z07) at the shear range of 0.02 . The influence on SPD shear capacity was very little when the shear angle was less than 0.02. With the increasing shear angle, obvious strain hardening was observed with the hysteresis curve in Figure 10, and the maximum normalized horizontal forces were 2.18 (at a shear angle of 0.04 for Rw04-z05), 1.89 (at a shear angle of 0.04 for Rw03-z03), 1.79 (at a shear angle of 0.034 for Rw03-z05), 1.75 (at a shear angle of 0.027 for Rw03-z06), 1.67 (at a shear angle of 0.017 ), 0.25 (at a shear angle of 0.08 for Flange). 


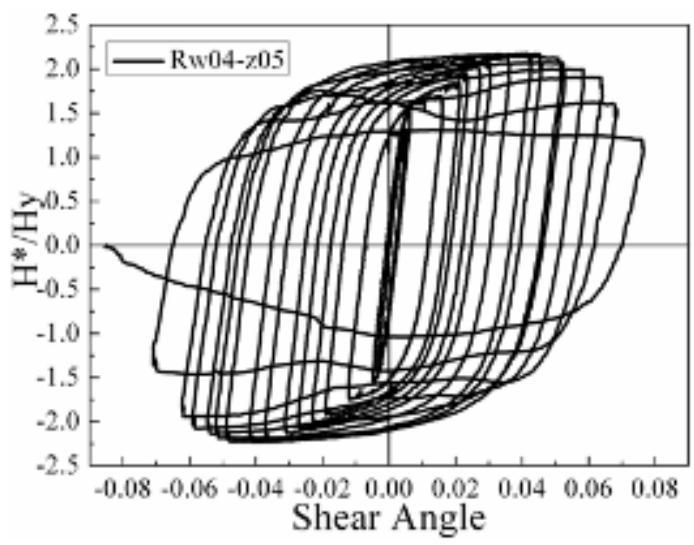

(a)

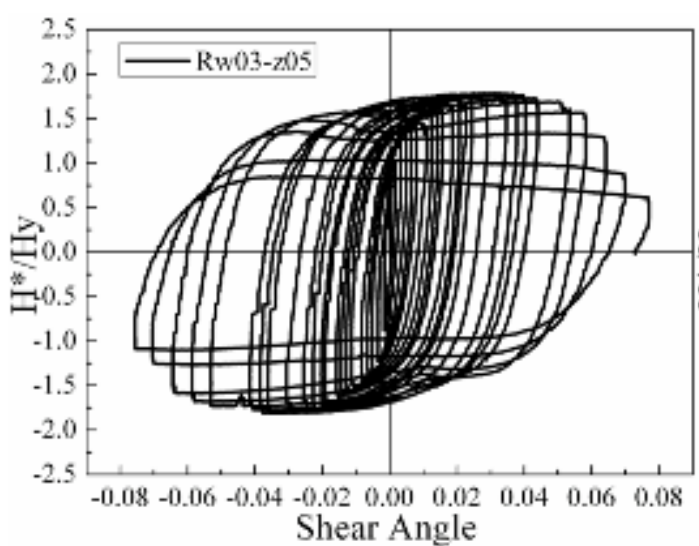

(c)

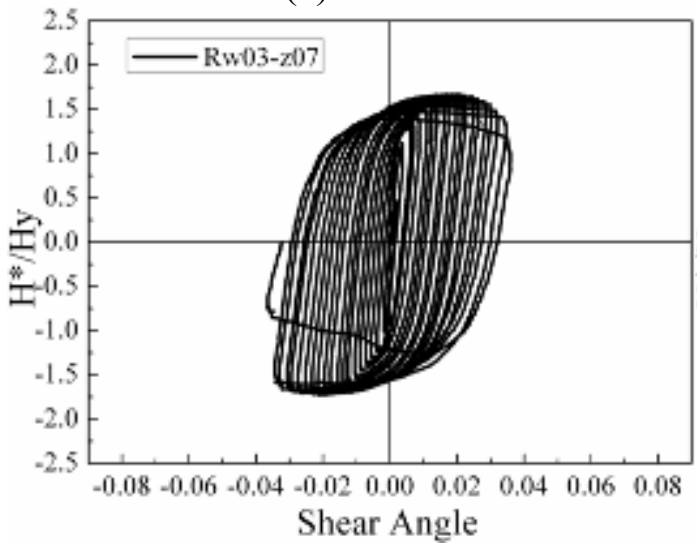

(e)

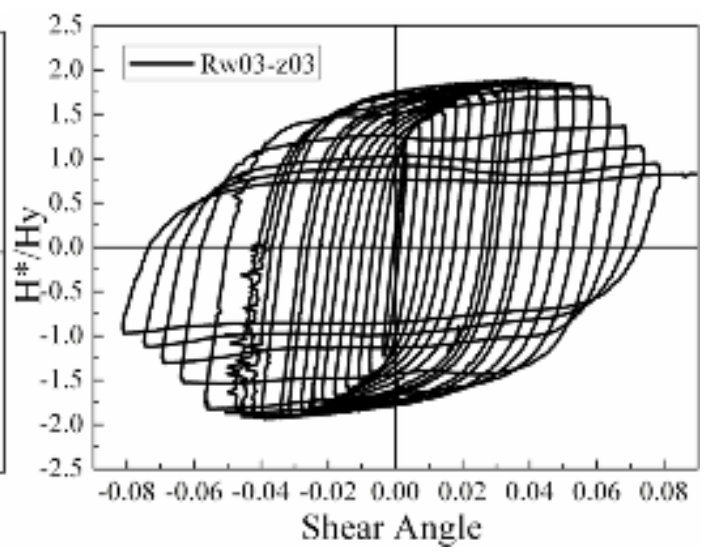

(b)

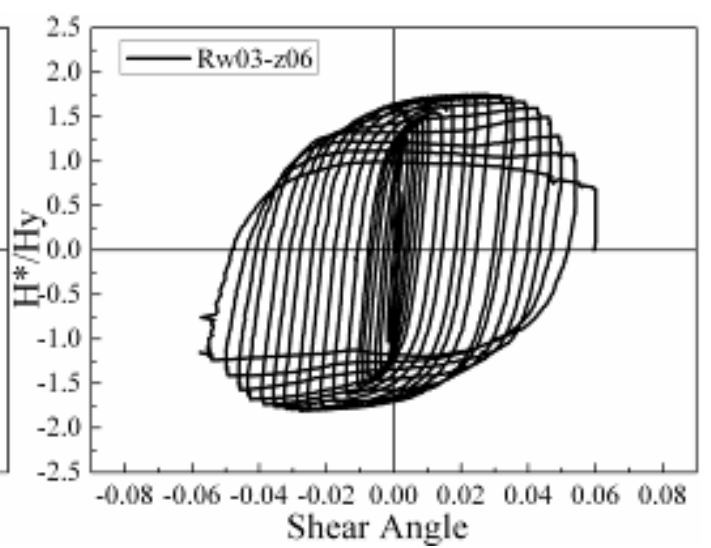

(d)

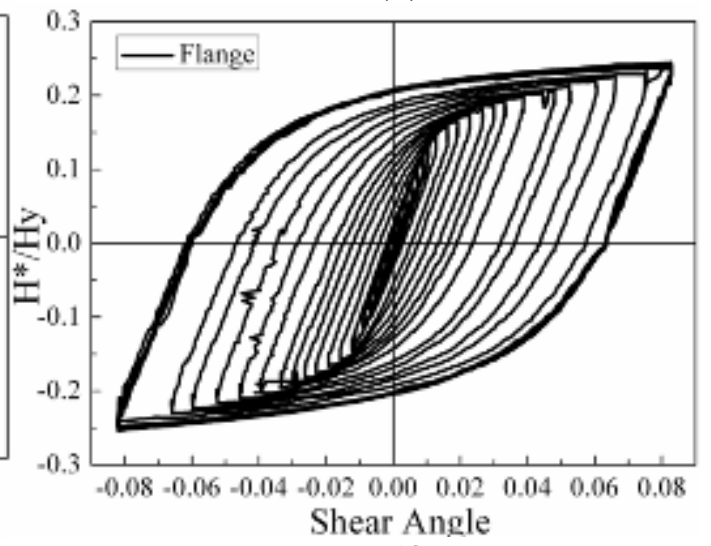

(f)

Figure 10. Horizontal Force Versus Shear Angle

It should be noted that Figure 10(f) presents the horizontal force versus shear angle relationship for the control specimen Flange (Figure 4). Here, $H^{*}$ is obtained in the same way of other specimens. To be consistent with the other plots in Figure 10, $H^{*}$ of the Flange is also normalized by divided by $H_{y}$. Here, $H_{y}$ is the yield shear force of Rw03 series of specimens $(=265.1 * 400 * 6=636.2 \mathrm{kN})$. Comparing the hysteresis curves between Flange and SPDs, it is clear that the frame-type specimen Flange opposes excellent ductility. The Flange is not only being the fix boundary of the web, but also contributes to the total horizontal resistance force. Take Rw03-z05 as an example. At a shear angle of 0.0355 , the peak $H^{*} / H_{y}$ value of Rw03-z05 is 1.53 . While the specimen Flange is about 0.18 at a shear angle of 0.0334 . Then, it might be roughly estimated that the shear force component resisted by flanges is about $11 \%$ of the total shear of an SPD. The contribution of flanges may reach more than this value, considering the interaction effect between the web and flanges. The 
conclusion is identical to the numerical results of Reference [9]. In their numerical analysis, the shear forces resisted by the flanges and the web were obtained separately. The shear force resisted by flanges was then about $13-20 \%$ of the total shear strength.

\subsection{Shear Force versus Shear Angle Relationship}

As shown in Figure 10, the normalized horizontal force was larger for a specimen with a smaller web thickness. This was due to the difference in the contribution of the flanges to the shear capacity among specimens with different web thickness. As discussed above, the flanges have a certain contribution to the total horizontal force. So the horizontal force sustained by the flanges must be subtracted if further understanding of the web shear capacity is needed. Figure 11 shows the relationship between normalized shear force $\left(H / H_{y}\right)$ versus shear angle, in which $H$ represents the shear force sustained only by the web. Since shear force sustained by the web or by the flanges cannot be measured separately from experiments, the shear force $H$ is given as the horizontal force $H^{*}$ subtracted by the resistance of the specimen Flange. This calculation method overestimates evidently the shear force of the web because it does not take account of the interaction between the web and the flanges. In addition, it should be noted that the two flanges for Specimen Rw04-z05 is $18 \mathrm{~mm}$ thick, not $24 \mathrm{~mm}$ as the Specimen Flange. Hence, the shear bearing force of $18 \mathrm{~mm}$-thick flange is firstly converted from $24 \mathrm{~mm}$-thick flange, according to the principle of equivalent area. Then the shear force $H$ sustained by the web of Rw04-z05 is calculated by subtracting the equivalent value from experimental results, $H^{*}$, of Rw04-z05.

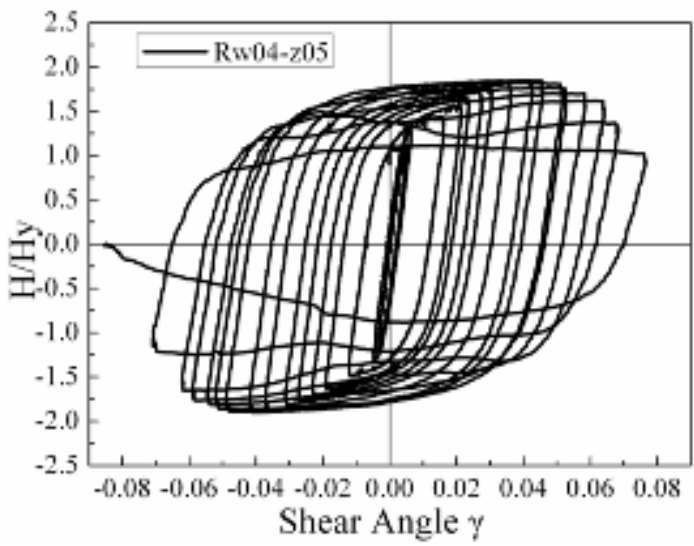

(a)

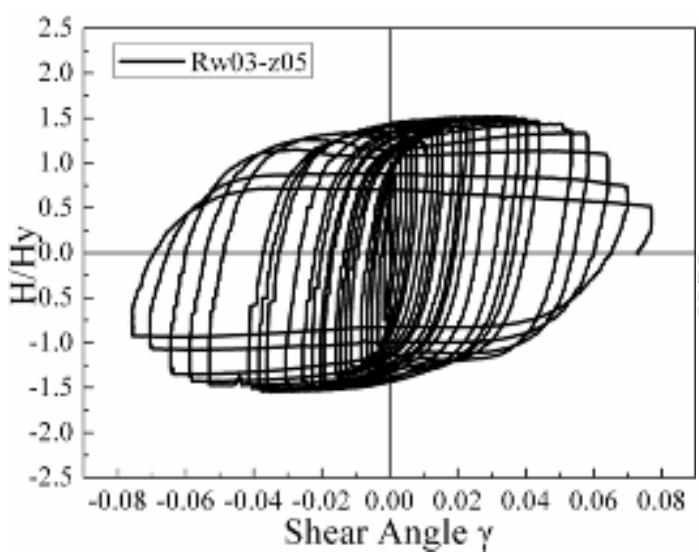

(c)

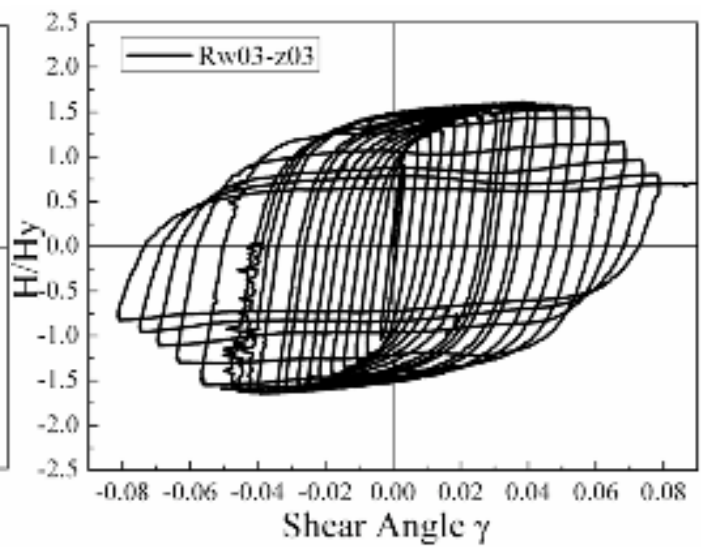

(b)

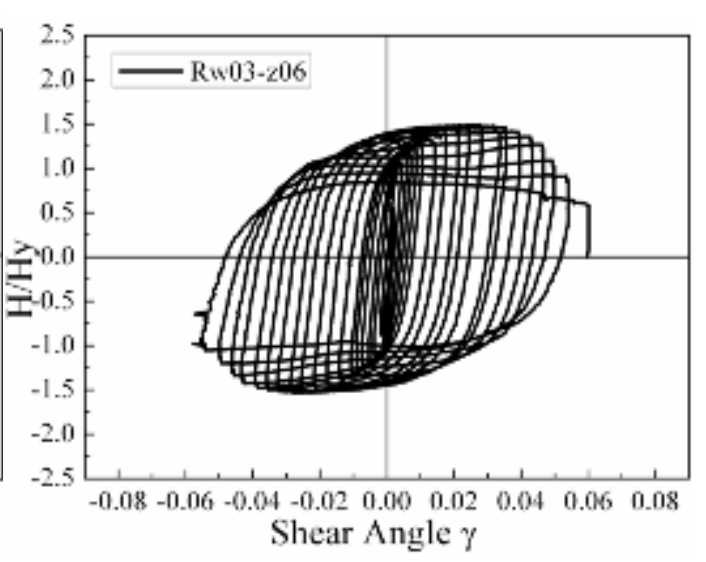

(d) 


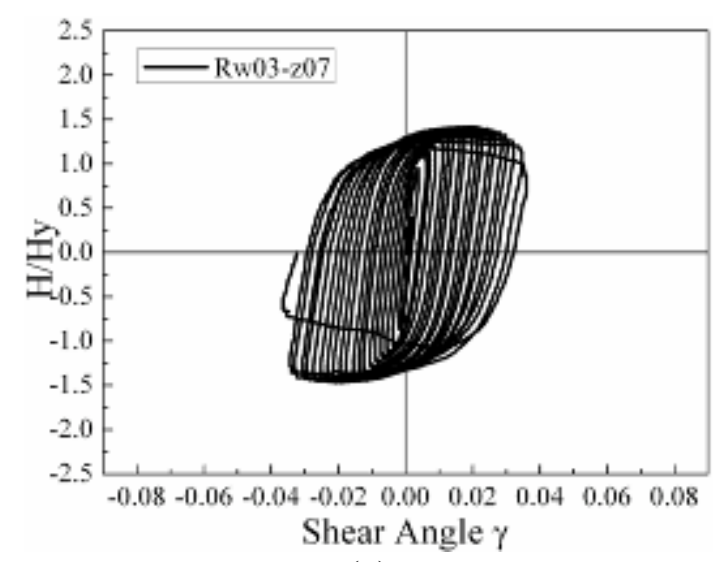

(e)

Figure 11. Shear Force Versus Shear Angle

To vividly describe the information included in the hysteresis curves, the envelopes of the curves are redrawn as shown in Figure 12. The envelope is obtained by plotting the peak shear angle of each cycle along with the corresponding normalized shear forces averaged from positive and negative loading sides. Discussions about the figures will be presented in the following section.

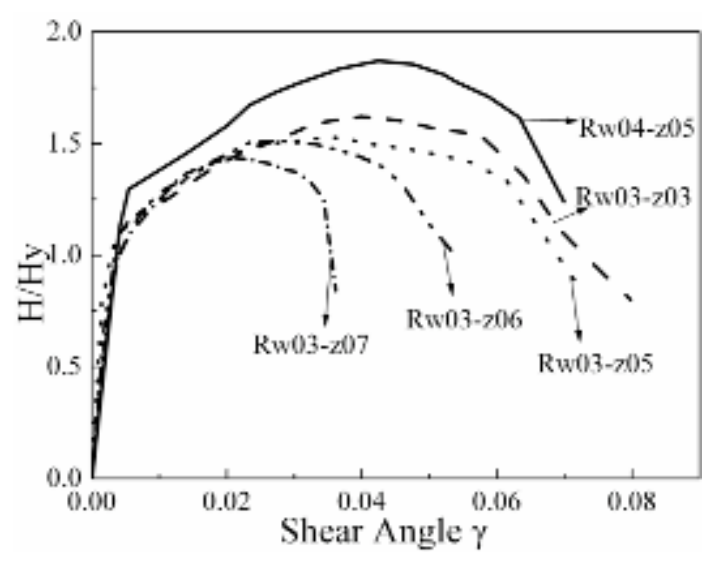

Figure 12. Envelopes of Shear Force Versus Shear Angle

\section{DISCUSSIONS AND COMMENTARY ON DESIGN}

According to the relationships between shear force and shear angle (Figure 11) and between the shear force envelope and shear angle (Figure 12) obtained from cyclic shear loading tests, some important points are discussed herein. These are: the range of shear deformation demand, the effect of axial compression ratio, web buckling, out-of-plane deformation, strain-hardening and energy dissipation behavior.

\subsection{Range of Shear Deformation Demand}

When evaluating the hysteretic behavior of dampers used as energy dissipation devices for an underground structural system, it is important to specify the range of shear deformation, which would be sustained under design loading. The range of deformation demand is also needed when developing hysteresis models for simulating the hysteresis behavior. Under the code for seismic design of subway structures in Shanghai, China [12], 1/250 is the maximum story-drift angle for moderate earthquakes (peak ground acceleration of $0.1 \mathrm{~g}$ ). As shown in Figure 1, assume inner 
columns are rigid enough (without deformation) and the horizontal deflection is totally absorbed by an SPD. Then the shear deformation demand would be calculated as $0.02(1 / 250 \times 3 / 0.6)$ for an SPD with the height of $0.6 \mathrm{~m}$ in a story which is commonly about $3 \mathrm{~m}$ in height. According to Figures 10 and 11, a shear angle of 0.02 can be reached except for the test specimen Rw03-z07. Its shear capacity began to decline when the shear angle exceeded 0.017 .

\subsection{The Effect of Axial Compression Ratio}

Figures 11 and 12 show that all specimens had excellent hysteretic behavior below a certain shear angle. When shear angle was less than 0.02 , the axial compression ratio had little influence on the performance of the damper. When the axial compression ratio was less than or equal to 0.5 , test specimens Rw04-z05, Rw03-z03 and Rw03-z05 revealed good ductility, and the maximum shear angle approached or exceeded 0.07 (more than $40 \gamma_{y}=0.002$ ). With the increasing axial compression ratio, the maximum shear angle was $0.052\left(26 \gamma_{y}\right)$ for Rw03-z06. When the axial pressure ratio reached 0.7 , the maximum shear angle rapidly decreased to 0.035 (about $17 \gamma_{y}$ ). In addition, increasing the axial compression ratio resulted in some decrease of shear capacity for the damper. It can be concluded that the shear panel dampers had good hysteresis performance when the shear angle was less than 0.02 , but that the ductility decreased significantly with an increasing axial compression ratio. Thus it is suggested that the axial compression ratio cannot be greater than 0.6 in the design and application of SPDs. The axial compression ratio of 0.6 can be considered as the critical ratio. When the ratio exceeds this critical value, the axial force will become the dominant factor in energy dissipation of SPDs. During design and application, it is recommended that a post-installation method be used. In this way, columns can withstand a great part of vertical loads while the damper mainly absorbs energy through horizontal shear loading.

\subsection{Web Buckling and Out-of-plane Deformation}

During the tests, initial web buckling was observed at a shear angle of 0.027 (Rw04-z05), 0.028 (Rw03-z03), 0.020 (Rw03-z05), 0.021 (Rw03-z06), and 0.012 (Rw03-z07). Web buckling did not lead to any sharp decrease in bearing capacity or energy dissipation capacity. The shear force continued to increase. Out-of-plane deformation started developing with increasing shear angle, and obvious out-of-plane deformation was observed at a shear angle of $0.045,0.042,0.029,0.028$, and 0.017 for Rw04-z05, Rw03-z03, Rw03-z05, Rw03-z06 and Rw03-z07, respectively. At the time, peak shear force was achieved, and no pinching (reduction in strength in the vicinity of zero displacement [2]) was observed. Subsequently, the shear strength began to decline, which was caused by larger out-of-plane deformation. From observing each specimen, it could be concluded that initial buckling did not have a significant impact on the shear force, and the shear force continued to increase with the development of out-of-plane deformation. When out-of-plane deformation became extremely obvious, the shear force reached its peak. With the increasing axial compression ratio, the shear angle capacity of shear panel dampers was reduced. When the axial load ratio reached 0.7, the shear angle capacity was less than half that of the ratio of 0.3 . It also indicated that if the axial compression ratio exceeded the critical axial compression ratio of 0.6 , the axial force would become the dominant factor in energy dissipation of shear panel dampers.

\subsection{Crack Influence}

As shown in Figure 9, a crack phenomenon could be observed from the test specimens. Tiny cracks happened in welded zones (between web, flange and stiffener) and local zones with large out-of-plane deformation. These soon developed into long cracks which meant the failure of the SPD. So the SPD should be checked in key positions in the process of using dampers or after an earthquake. 


\subsection{Strain-hardening Behavior}

Figures 11 and 12 show that when the shear angle was less than a certain angle (about 0.02), obvious isotropic hardening behavior was observed. However, this changed to combined isotropic and kinematic hardening behavior at shear angles from 0.02 to 0.04 .

\subsection{Energy Dissipation Capacity}

Energy dissipation capacity is very important in evaluating the performance of SPDs as energy dissipation devices. Here, dissipated energy, $E_{\mathrm{i}}$, is normalized by $E_{\mathrm{e}}$, defined as $H_{y} \Delta_{y} / 2$. The normalized dissipated energy, $E_{i} / E_{e}$, is calculated for each half-cycle as shown in Figure 13 . The cumulative dissipated energy is defined as the sum of previous dissipated energy, $\Sigma E_{i} / E_{e}$. The cumulative dissipated energy versus cumulative shear angle at each half cycle is shown in Figure 14. It indicates that the maximum cumulative dissipated energy and cumulative shear angle decreased with an increasing axial compression ratio. For axial compression ratios of 0.6 and 0.7 , the maximum cumulative energy was about half that of a ratio of 0.3 . But in the range of the shear deformation demand (with a cumulative shear angle of about 0.4), cumulative dissipated energy had little difference among the specimens, as shown in Figure 14(a). Thus, when considering the axial compression ratio, the range of shear deformation demand also should be taken as an important factor in the design of SPDs.

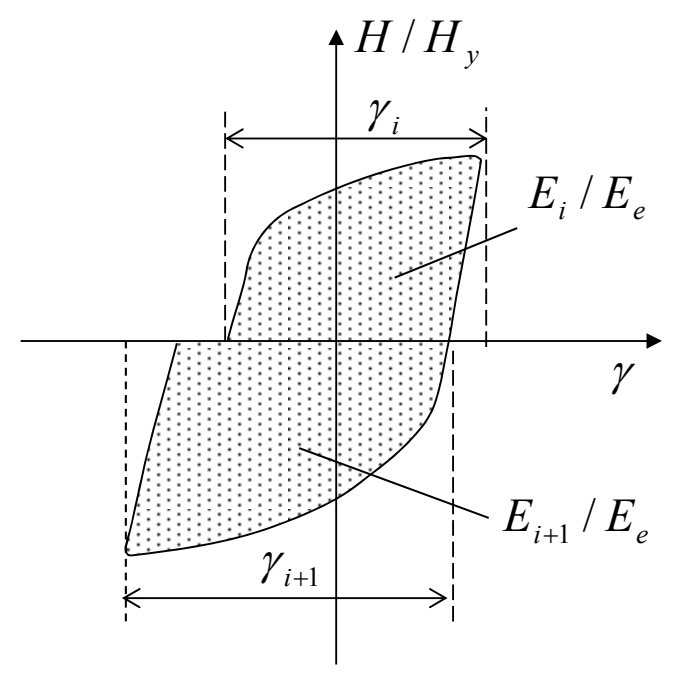

Figure 13. Definition of Dissipated Energy in Each Half Cycle, $E_{i} / E_{e}$

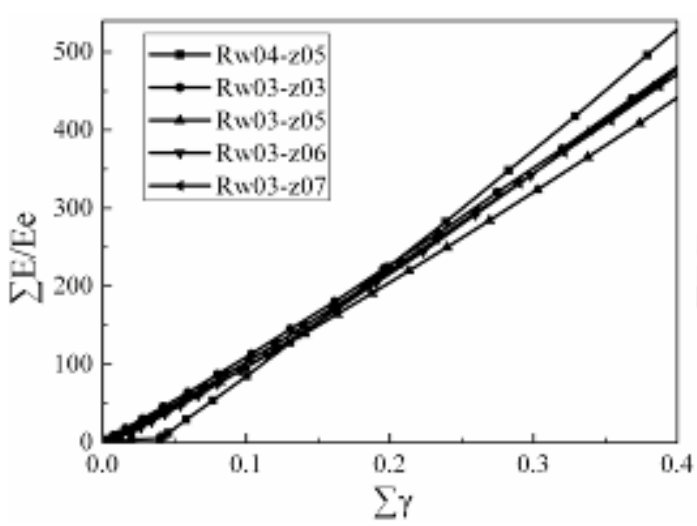

(a)

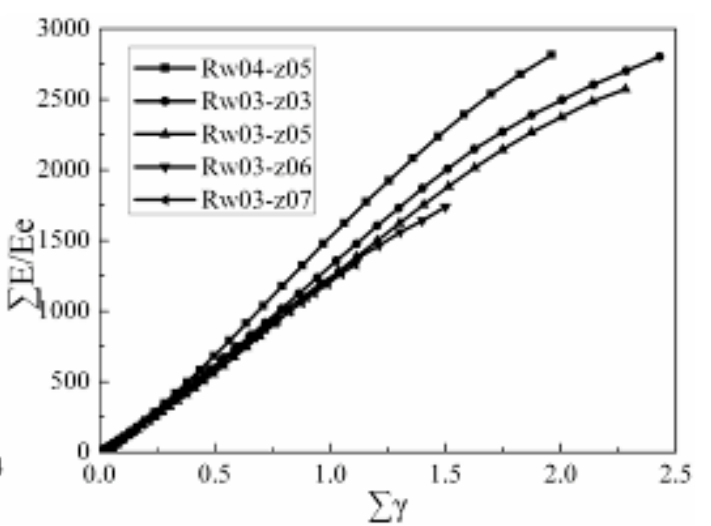

(b)

Figure 14. Cumulative Dissipated Energy versus Cumulative Shear Angle 


\section{CONCLUSIONS}

This paper has presented an experimental study examining the hysteretic behavior of shear panel dampers made of mild steel Q235B. Regarding the axial compression ratio as the most important control parameter, shear capacity, web buckling, hysteretic behavior and energy dissipation behavior were researched. A summary of findings is as follows:

1) With an increasing axial compression ratio, the shear capacity and ductility of SPDs decreased. When the axial compression ratio equals 0.7 , the bearing capacity, ductility and energy dissipation capacity of an SPD decrease sharply. In the case of 0.6 , the ductility is slightly affected while its bearing capacity remains stable. So 0.6 can be regarded as the critical axial compression ratio in the design and application of SPDs.

2) From comparisons between SPDs and the control group (Flange), the contribution of flanges of an SPD to resisting shear loading was about $15 \%$.

3) Obvious strain-hardening behavior was observed in the test and no pinching phenomenon occurred in hysteretic curves. With increasing shear angle, isotropic hardening behavior gradually changed to kinematic hardening behavior.

4) When the shear angle is less than 0.02, the shear panel dampers have good hysteresis performance. However, with an increasing axial compression ratio, the ductility and energy dissipation decreased significantly.

\section{ACKNOWLEDGMENTS}

This research was supported by the National Natural Science Foundation of China (Grant No. 50908169), National Basic Research Program of China (973 Program: 2011CB013600), State Key Laboratory of Geo-Hazard Prevention and Geo-Environment Protection (Grant No. SKLGP2011K009), Program for Changjiang Scholars and Innovative Research Team in University (PCSIRT, IRT1029), the Fundamental Research Funds for the Central Universities and the Kwang-Hua Fund for College of Civil Engineering, Tongji University. All supports are gratefully acknowledged.

\section{REFERENCES}

[1] Nakashima, M., Iwai, S., Iwata, M., Takeuchi, T., Konomi, S., Akazawa, T. and Saburi, K., "Energy Dissipation Behavior of Shear Panels Made of Low Yield Steel", Earthquake Engineering and Structural Dynamics, 1994, Vol. 23, No. 12, pp. 1299-1313.

[2] Nakashima, M., "Strain-hardening Behavior of Shear Panels Made of Low-yield Steel.1: Test", Journal of Structural Engineering, 1995, Vol. 121, No. 12, pp. 1742-1749.

[3] Nakashima, M. Akazawa, T. and Tsuji, B., "Strain-hardening Behavior of Shear Panels Made of Low-yield Steel. 2: Model", Journal of Structural Engineering, 1995, Vol. 121, No. 12, pp. 1750- 1757.

[4] Chen, S.J. and Kuo, C.L., "Experimental Study of Vierendeel Frames with LYP Steel Shear Panels", Steel Structures, 2004, Vol. 4, pp. 179-186.

[5] Chen Z.Y., Ge H.B. and Usami T., "Study on Seismic Performance Upgrading for Steel Bridge Structures by Introducing Energy-dissipation Members", Journal of Structural Engineering, JSCE, 2007, Vol. 53, pp. 540-549.

[6] Huo, H., Bobet, A., Fernández, G. and Ramírez, J., "Load Transfer Mechanisms Between Underground Structure and Surrounding Ground: Evaluation of the Failure of the Daikai Station”, Journal of Geotechnical and Geoenvironmental Engineering, 2005, Vol. 131, No. 
12, pp. 1522-1533.

[7] Gustavo, J.P.M., Bobet, A. and Ramirez, J., "Evaluation of Soil-structure Interaction and Structural Collapse in Daikai Subway Station during Kobe Earthquake", ACI Structural Journal, 2006, Vol. 103, No. 1, pp. 113-122.

[8] Mansour, N., Christopoulos, C. and Tremblay, R., "Experimental Validation of Replaceable Shear Links for Eccentrically Braced Steel Frames", Journal of Structural Engineering, 2011, Vol. 137, No. 10, pp. 1141-1152.

[9] Chen, Z.Y., Ge, H.B. and Usami, T., "Hysteretic Model of Stiffened Shear Panel Dampers", Journal of Structural Engineering, 2006, Vol. 132, No. 3, pp. 478-483.

[10] Chen, Z.Y., Ge, H.B., Kasai, A. and Usami, T., "Simplified Seismic Design Approach for Steel Portal Frame Piers with Hysteretic Dampers", Earthq. Eng. Struct. Dyn, 2007, Vol. 36, No. 4, pp. 541-562.

[11] Chen, Z.Y., Ge, H.B., Kasai, A. and Usami, T., "Numerical Study on Development of Hysteretic Model for Stiffened Steel Shear Panel Dampers", J. Struct. Eng., JSCE, 2006, Vol. 52, pp. 573-582.

[12] DG/TJ08-2064-2009, “Code for Seismic Design of Subway Structures”, Shanghai Urban Constructions Communications, Shanghai, 2010. (in Chinese) 